\title{
Modified Reduced Neighborhood Topological Indices of Cycloalkanes, Clycloalkenes, and Cycloalkynes
}

\author{
Sophia Shalini G.B. ${ }^{1, *}$, Dhananjayamurthy B.V. ${ }^{2}$ \\ Department of Mathematics, Mount Carmel College, Bangalore-58, India; sofi.saranya920@gmail.com (S.S.G.B.); \\ 2 Department of Mathematics, Nitte Meenakshi Institute of Technology, Yelahanka, Bangalore-64; \\ dhananjayamurthy.bv@nmit.ac.in (D.B.V.); \\ * Correspondence: sofi.saranya920@gmail.com (S.S.G.B.);
}

Scopus Author ID 57221272110

Received: 14.07.2021; Revised: 29.08.2021; Accepted: 3.09.2021; Published: 18.10.2021

\begin{abstract}
In this paper, some newly introduced topological indices, namely, modified reduced neighborhood first Zagreb index $\left(R N M_{1}^{*}(G)\right)$ and modified forgotten reduced neighborhood index $\left(R N M_{3}^{*}(G)\right)$ of a graph, $G$ are used to establish the general formula for the molecular graphs of cycloalkanes, cycloalkenes, and cycloalkynes. We also calculate the exact values of some special types of these molecular graphs by attaching the alkyls instead of hydrogen atoms. Also, the QSPR analysis for these newly introduced indices is studied, and we exhibit a good correlation with characteristics on these molecular graphs.
\end{abstract}

Keywords: modified reduced neighborhood first Zagreb index; modified forgotten reduced neighborhood index; cycloalkanes; cycloalkenes; cycloalkynes.

(C) 2021 by the authors. This article is an open-access article distributed under the terms and conditions of the Creative Commons Attribution (CC BY) license (https://creativecommons.org/licenses/by/4.0/).

\section{Introduction}

Let $G=(V, E)$ be a simple connected graph with vertex set $V(G)$ and an edge set $E(G)$. A chemical graph is a graph obtained by replacing the atoms by vertices and bonds by edges. The connection between the molecules and their respective graphs is a part of Mathematical Chemistry which is predominant for the growth of Molecular Chemistry and QSAR/QSPR investigations. In molecular graph theory, a numeric value corresponding to a structurally invariant graph is known as the topological index. The most studied topological indices are the first and second Zagreb indices and have many applications in molecular graph theory. The Zagreb indices are important in the theory of the total $\pi$-electron energy of alternant hydrocarbons. Gutman and Trinajstic introduced the first and second Zagreb indices in 1972 [1] and defined respectively as,

$$
M_{1}(G)=\sum_{v_{i} \in V(G)} d_{G}\left(v_{i}\right)^{2} \text { and } \quad M_{2}(G)=\sum_{v_{i} v_{j} \in E(G)} d_{G}\left(v_{i}\right) d_{G}\left(v_{j}\right)
$$

For all the recent studies on Zagreb indices and the mathematical properties, we refer [2-6]. For chemical applications of Zagreb indices, refer [7-11]. Sourav Mondal, Nilanjan De, Anita Pal investigated the degree-based and neighborhood degree sum-based topological indices for some of the antiviral drugs by using the polynomial approach [12]. Anwar Saleh, Sophia Shalini G B, and Dhananjayamurthy B V studied the reduced neighborhood topological indices and $R N M$ - polynomial for the treatment of COIVID-19 [13]. They have also defined modified reduced neighborhood first Zagreb index and modified forgotten reduced 
neighborhood index of a graph $G$. This paper uses these two topological indices to establish the general formula for the molecular graphs of cycloalkanes, cycloalkenes, and cycloalkynes.

Cycloalkanes are cyclic hydrocarbons, implying that the carbons of the atom are arranged as a ring. Cycloalkanes are likewise immersed, implying that the entirety of the carbons molecules that make up the ring are single attached to different particles (no twofold or triple bonds). To clarify it all the more obviously, the cycloalkane is made up with only hydrogen and carbon molecules which is randomly distributed in a framework with just a single ring and single carbon bonds with the general formula $C_{n} H_{2 n}$. Basically, cycloalkanes share comparable general actual properties and characteristics with alkanes. In any case, it has some extraordinary or various properties. To delineate, the edge of boiling over and softening mark of cycloalkanes are higher than the basic alkanes. Also, it has a high calorific worth and a low edge of freezing over. The impact of cycloalkane is chiefly to be utilized to deliver fuel in the applications. In naphthenes, the most well-known cycloalkanes applied in the business might be cyclohexane, a lackluster fluid, which is not difficult to unstable and consume. Flammable gas and biogas contain a parcel of methane which has been utilized widely lately as spotless energy, and it is very famous these days. Subsequently, it can likewise be utilized in greases and black-top. Besides being a significant synthetic crude material, cycloalkanes have been generally utilized in the medication field, and alcohols are customarily considered the fundamental element of numerous significant drugs [14].

Cycloalkenes are unsaturated hydrocarbons having a couple of hydrogen atoms and a couple of bonds with the general formula $\mathrm{C}_{n} \mathrm{H}_{2 n-2}$. In contrast, to open chain alkenes, cycloalkenes have fewer hydrogen atoms and fewer limits. So there is less intermolecular energy inside the particle. The significant properties of cycloalkenes are as follows: at room temperature, the vast majority of them are fluid despite having extremely low dissolvability in water. Be that as it may, the initial not many cycloalkenes are in a vaporous state and are once in a while strong at room temperature. The cycloalkenes show comparative properties as cycloalkanes; however, a contrast in the way that cycloalkenes have, at any rate, one double bond [15].

Cycloalkyne is the cyclic simple of an alkyne with the general formula $C_{n} H_{2 n-4}$. Cycloalkyne comprises a closed ring of carbon molecules containing at least one triple bond. Enormous alkyne-containing carbocycles might be unstrained, while the littlest constituents of this class of particles may encounter such an excess of strain that they still can't seem to be noticed tentatively. Cyclooctyne is the smallest cycloalkyne equipped for being confined and put away as a steady compound. In spite of this, more modest cycloalkynes can be created and caught through responses with other natural atoms or through complexation to change metals [16]. For more details on cycloalkanes, cycloalkenes, and cycloalkynes, we refer to [17-22]. Figure 1 represents the molecular graphs of cycloalkanes, cycloalkenes, and cycloalkynes, respectively.

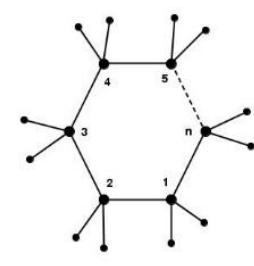

a) cycloalkanes

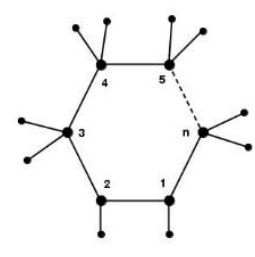

b) cycloalkenes

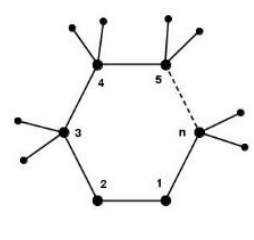

c) cycloalkynes

Figure 1. The molecular graphs of Cycloalkanes, Cycloalkenes, and Cycloalkynes. 
The group of alkyl or the parts of alkyl denoted by $R_{j}, j \in Z^{+}$are the classes of alkanes with one hydrogen atom eliminated. Here $R_{1}, R_{2}, R_{3}, \ldots$ represents methyl, ethyl, propyl, and so on, respectively, as shown in Figure 2.

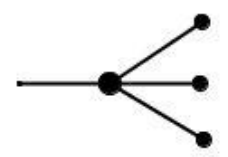

$\mathrm{R}_{1}$ :Methyl

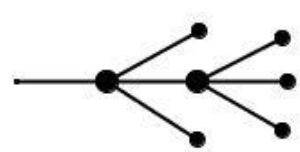

$R_{2}$ :Ethyl

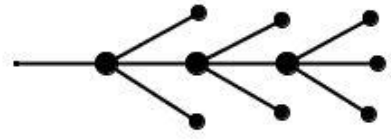

$R_{3}:$ Propyl

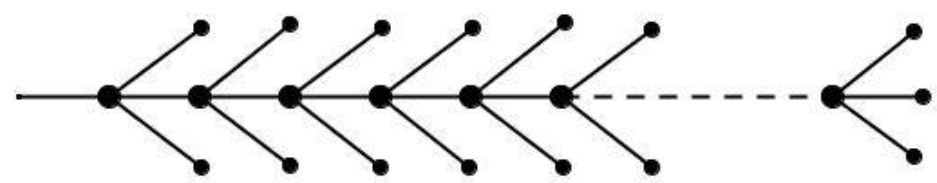

$\mathbf{R}_{\mathbf{j}}$ :alkyl

Figure 2. Molecular graph of alkyls.

In the next section, we shall discuss the modified, reduced topological indices of the special kind of cycloalkanes, cycloalkenes, and cycloalkenes, which are obtained by replacing each hydrogen atom of the molecular graph by attaching the alkyl $R_{j}$ denoted by $C_{n}^{R_{j}}$. For our convenience, we denote this special family of cycloalkanes, cycloalkenes, and cycloalkynes by $C_{n}^{R_{k}}, C_{n}^{R_{m}}$ and $C_{n}^{R_{t}}$ respectively. Figure 3 represents the molecular graph of the special kind of cycloalkanes denoted by $C_{n}^{R_{k}}$.

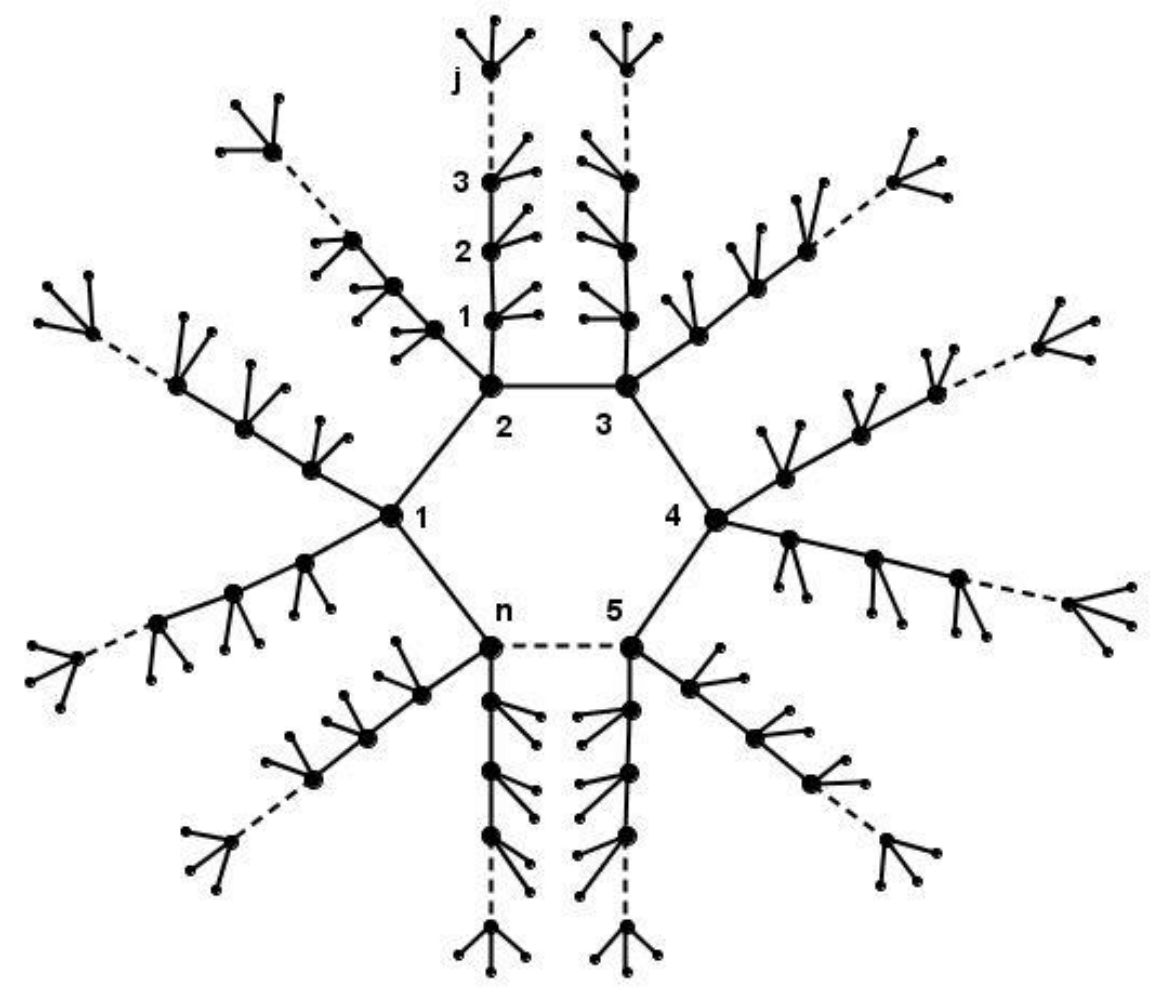

Figure 3. Molecular graph of $C_{n}^{R_{k}}$. 


\section{Materials and Methods}

Our main results include using the concept of modified, reduced neighborhood first Zagreb index and modified forgotten reduced neighborhood index of a graph $G$ and establish the general formula for the molecular graphs of cycloalkanes, cycloalkenes, and cycloalkynes and calculate the exact values of some special types of these molecular graphs by attaching the alkyls instead of hydrogen atoms. Also, the QSPR analysis for these newly introduced indices is studied, and using regression analysis, we exhibit a good correlation with characteristics on these molecular graphs.

\section{Results and Discussion}

This section has been divided into two subsections with the definitions and computational results.

\subsection{Modified reduced neighborhood topological indices.}

Definition 3.1.1.[13].

Let $G=(V, E)$ be a simple graph with $|V(G)|=n$ and $|E(G)|=n$. The $N$-degree of a vertex $u$ is defined as the sum of the degree of the vertices in the open neighborhood of $u$. That is,

$$
d_{N}(u)=\sum_{v \in N(u)} d(v)
$$

The reduced neighborhood degree of a vertex $u$ is defined as

$$
d_{N \prime}(u)=\sum_{v \in N(u)}(d(v)-1) .
$$

Definition 3.1.2.[13].

Let $G=(V, E)$ be a simple graph with $|V(G)|=n$ and $|E(G)|=n$. Then the reduced neighborhood first Zagreb index and second Zagreb index denoted by $R N M_{1}(G)$ and $R M_{2}(G)$ respectively, are defined as;

$$
R N M_{1}(G)=\sum_{u \in V(G)} d_{N^{\prime}}(u)^{2} \text { and } R N M_{2}(G)=\sum_{u v \in E(G)} d_{N^{\prime}}(u) d_{N^{\prime}}(v) .
$$

Definition 3.1.3.[13].

The modified, reduced neighborhood version of the first Zagreb index denoted by $\operatorname{RNM}_{1}^{*}(G)$ is defined as:

$$
R N M_{1}^{*}(G)=\sum_{u v \in E(G)}\left[d_{N^{\prime}}(u)+d_{N^{\prime}}(v)\right] .
$$

Definition 3.1.4.[13].

The modified, reduced neighborhood version of the forgotten topological index denoted by $\operatorname{RNM}_{3}^{*}(G)$ is defined as:

$$
R N M_{3}^{*}(G)=\sum_{u v \in E(G)}\left[d_{N^{\prime}}(u)^{2}+d_{N^{\prime}}(v)^{2}\right]
$$


Definition 3.1.5.

For any graph $G$, we define the set of different types of edges as

$R_{(a, b)}(G)=\left\{u v \in E(G): d_{N^{\prime}}(u)=a\right.$ and $\left.d_{N^{\prime}}(v)=b\right\}$.

Theorem 1 . Let $G \cong C_{n}^{2 n}$ with $n \geq 3$ be a molecular graph of cycloalkane. Then

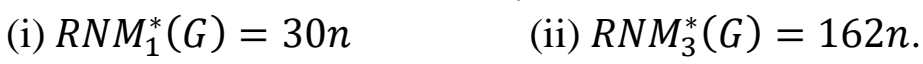

Proof. Let $G \cong C_{n}^{2 n}$ be a molecular graph of cycloalkane with $n \geq 3$ vertices. Then $G$ has $3 n$ vertices. Out of these vertices, there are $n$ vertices of reduced neighborhood degree 6 and $2 n$ vertices of reduced neighborhood degree 3 . The edge partition of $G$ is $\left|R_{(6,6)}\right|=n$ and $\left|R_{(6,3)}\right|=2 n$. Thus,

$$
\begin{aligned}
\operatorname{RNM}_{1}^{*}(G) & =\sum_{u v \in E(G)}\left[d_{N^{\prime}}(u)+d_{N^{\prime}}(v)\right] \\
& =12 n+18 n=30 n . \\
\operatorname{RNM}_{3}^{*}(G) & =\sum_{u v \in E(G)}\left[d_{N^{\prime}}(u)^{2}+d_{N^{\prime}}(v)^{2}\right] \\
= & 72 n+90 n=162 n .
\end{aligned}
$$

Theorem 2. Let $G \cong C_{n}^{2 n-2}$ with $n \geq 3$ be a molecular graph of cycloalkene. Then

(i) $R N M_{1}^{*}(G)= \begin{cases}56, & \text { if } n=3 \\ 30 n-34, & \text { if } n>3\end{cases}$

(ii) $\operatorname{RNM}_{3}^{*}(G)=\left\{\begin{array}{cc}240, & \text { if } n=3 ; \\ 2(81 n-127), & \text { if } n>3 ;\end{array}\right.$

Proof. Case 1: Let $n=3$.

Let $G \cong C_{n}^{2 n-2}$ be a molecular graph of cycloalkene with $n=3$ vertices. Then there are 2 vertices of reduced neighborhood degree 5, one vertex of reduced neighborhood degree 4 , two pendant vertices of reduced neighborhood degree 2 , and two pendant vertices of reduced neighborhood degree 3. The edge partition is $\left|R_{(4,5)}\right|=2,\left|R_{(5,5)}\right|=1,\left|R_{(5,2)}\right|=2$ and $\left|R_{(4,3)}\right|=2$. Thus,

$$
\begin{aligned}
R N M_{1}^{*}(G) & =\sum_{u v \in E(G)}\left[d_{N^{\prime}}(u)+d_{N^{\prime}}(v)\right] \\
& =2(9)+10+2(7)+2(7)=56 . \\
R N M_{3}^{*}(G) & =\sum_{u v \in E(G)}\left[d_{N^{\prime}}(u)^{2}+d_{N^{\prime}}(v)^{2}\right] \\
& =2(41)+50+2(29)+2(25)=240 .
\end{aligned}
$$

Case 2: Let $n>3$. 
Let $G \cong C_{n}^{2 n-2}$ be a molecular graph of cycloalkene with $n \geq 4$ vertices. $G$ has $3 n-2$ vertices. Out of these vertices, there are 4 vertices of reduced neighborhood degree $5, n-4$ vertices of reduced neighborhood degree 6 together which corresponds to the carbon atoms of the cycloalkanes. In the remaining $2 n-2$ pendant vertices, there are two vertices of reduced neighborhood degree 2 and $2 n-4$ vertices of reduced neighborhood degree 3 . The edge partition is $\left|R_{(5,5)}\right|=3,\left|R_{(5,6)}\right|=2,\left|R_{(6,6)}\right|=n-5,\left|R_{(2,5)}\right|=2,\left|R_{(3,5)}\right|=4$ and $\left|R_{(3,6)}\right|=$ $2(n-4)$. Thus,

$$
\begin{aligned}
R N M_{1}^{*}(G) & =\sum_{u v \in E(G)}\left[d_{N^{\prime}}(u)+d_{N^{\prime}}(v)\right] \\
& =3(10)+2(11)+(n-5)(12)+2(7)+4(8)+2(n-4)(9) \\
& =30 n-34 . \\
R N M_{3}^{*}(G) & =\sum_{u v \in E(G)}\left[d_{N^{\prime}}(u)^{2}+d_{N^{\prime}}(v)^{2}\right] \\
= & 3(50)+2(61)+(n-5)(72)+2(29)+4(34)+2(n-4)(45) \\
= & 2(81 n-127) .
\end{aligned}
$$

Theorem 3. Let $G \cong C_{n}^{2 n-4}$ with $n \geq 5$ be a molecular graph of cycloalkyne. Then

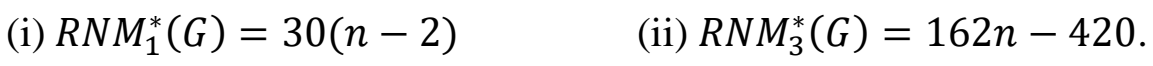

Proof. Let $G \cong C_{n}^{2 n-4}$ be a molecular graph of cycloalkyne with $n \geq 5$ vertices. Then $G$ has $3 n-4$ vertices. Out of these vertices, there are 4 vertices of reduced neighborhood degree $4, n-4$ vertices of reduced neighborhood degree 6 , and $2 n-4$ vertices of reduced neighborhood degree 3. The edge partition of $G$ is $\left|R_{(4,4)}\right|=3,\left|R_{(4,6)}\right|=2,\left|R_{(6,6)}\right|=n-$ $5,\left|R_{(4,3)}\right|=4$ and $\left|R_{(6,3)}\right|=2 n-8$. Thus,

$$
\begin{aligned}
R N M_{1}^{*}(G) & =\sum_{u v \in E(G)}\left[d_{N^{\prime}}(u)+d_{N^{\prime}}(v)\right] \\
= & 24+20+12(n-5)+9(2 n-8)+28=30(n-2) . \\
\operatorname{RNM}_{3}^{*}(G) & =\sum_{u v \in E(G)}\left[d_{N^{\prime}}(u)^{2}+d_{N^{\prime}}(v)^{2}\right] \\
& =96+104+(n-5) 72+(2 n-8) 45+100=162 n-420 .
\end{aligned}
$$

Theorem 4. Let $G \cong C_{n}^{R_{k}}$ be a molecular graph of a special type of cycloalkane with $n \geq 3$ and $k \geq 2$ vertices. Then
(i) $\operatorname{RNM}_{1}^{*}(G)=30 n(2 k+1)$
(ii) $\operatorname{RNM}_{3}^{*}(G)=324 n k+378 n$.

Proof. Let $G \cong C_{n}^{R_{k}}$ be a molecular graph of a special type of cycloalkane, as shown in Figure 3. Then the graph $G$ has $6 n k+3 n$ vertices, of which $n$ of them represent the vertices of a cycle, and $2 n(3 k+1)$ represent the vertices of alkyls. Out of these vertices, there are $n$ vertices of reduced neighborhood degree $12,2 n(k-1)$ vertices of reduced neighborhood 
degree 6 , and $2 n(2 k+2)$ vertices of reduced neighborhood degree 3 . The edge partition of $G$ is $\left|R_{(12,12)}\right|=n,\left|R_{(12,6)}\right|=2 n,\left|R_{(6,6)}\right|=2 n(k-2),\left|R_{(6,3)}\right|=2 n(2 k-1)$ and $\left|R_{(3,3)}\right|=6 n$. Thus,

$$
\begin{aligned}
R N M_{1}^{*}(G) & =\sum_{u v \in E(G)}\left[d_{N^{\prime}}(u)+d_{N^{\prime}}(v)\right] \\
& =24 n+36 n+24 n(k-2)+18 n(2 k-1)+36 n=30 n(2 k+1) \\
\operatorname{RNM}_{3}^{*}(G) & =\sum_{u v \in E(G)}\left[d_{N^{\prime}}(u)^{2}+d_{N^{\prime}}(v)^{2}\right] \\
& =288 n+360 n+2 n(k-2)(72)+2 n(2 k-1)(45)+108 n \\
& =324 n k+378 n .
\end{aligned}
$$

Theorem 5. Let $G \cong C_{n}^{R_{m}}$ be a molecular graph of a special type of cycloalkene with $n \geq 5$ and $m \geq 3$ vertices. Then

(i) $\operatorname{RNM}_{1}^{*}(G)=60 \mathrm{~nm}+30 \mathrm{n}-60 \mathrm{~m}-46$.

(ii) $\operatorname{RNM}_{3}^{*}(G)=324 n m+378 n-324 m-842$.

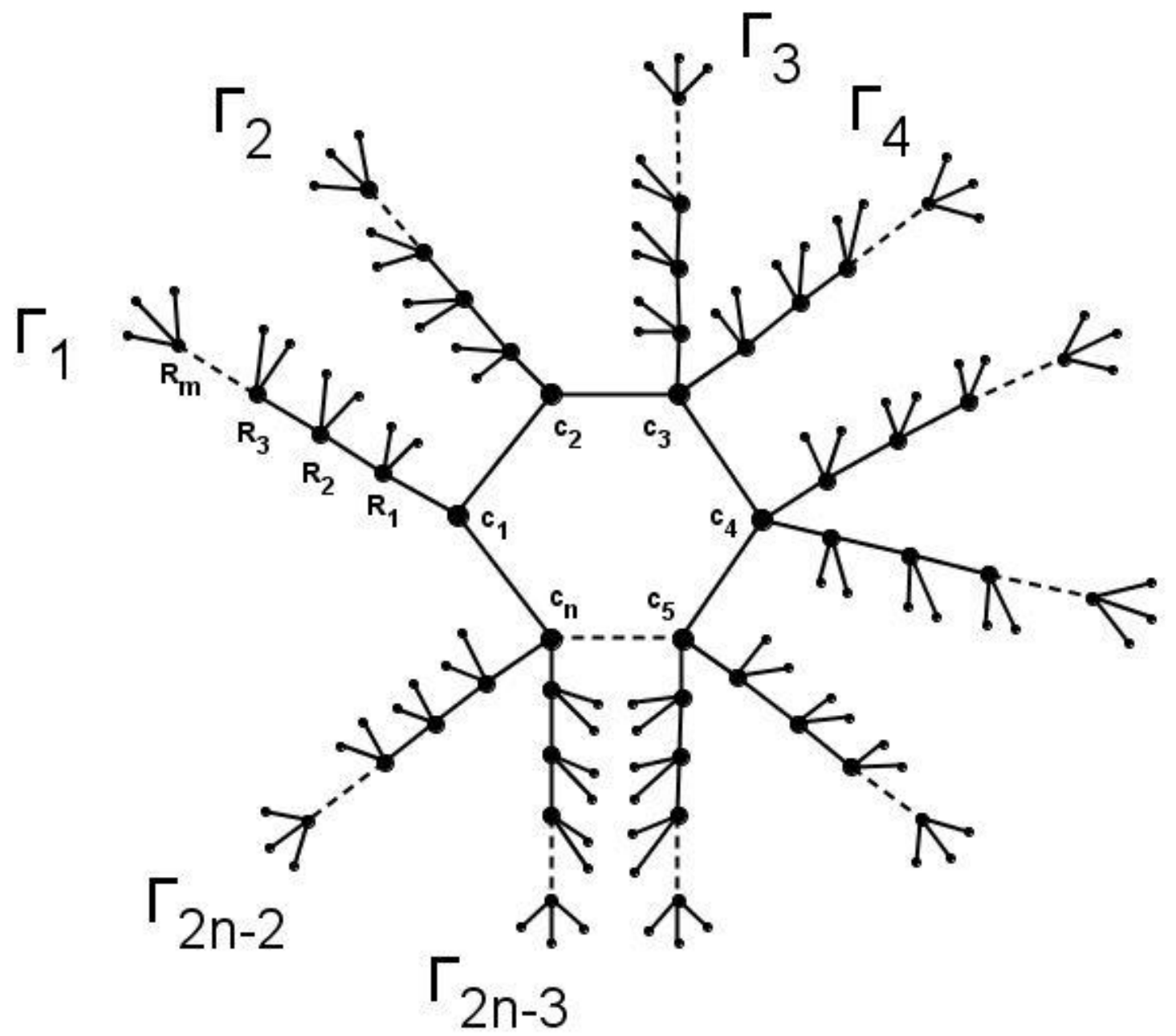

Figure 4. Molecular graph of $C_{n}^{R_{m}}$. 
Proof. Let $G \cong C_{n}^{R_{m}}$ be a molecular graph of a special type of cycloalkene as shown in Figure 4. Then the graph $G$ has $6 n m+3 n-6 m-2$ vertices of which $n$ of them represent the vertices of a cycle and $(2 n-2)(3 m+1)$ represent the vertices of alkyls. Out of these vertices, there are 2 vertices of reduced neighborhood degree 8,2 vertices of reduced neighborhood degree 11 , and $n-4$ vertices of reduced neighborhood degree 12 together, which corresponds to the vertices of a cycle. In the remaining $(2 n-2)(3 m+1)$ vertices of alkyls, there are two vertices of reduced neighborhood degree $5,2(m-2)+(2 n-4)(m-$ 1) vertices of reduced neighborhood degree $6,2 n-2$ vertices of reduced neighborhood degree 3 and $2(2 m+1)+(2 n-4)(2 m+1)$ vertices of reduced neighborhood degree 3 . Let $\Gamma_{1}, \Gamma_{2}, \ldots \ldots, \Gamma_{(2 n-2)}$ represent each alkyl group $R_{m}$ attached to the cycle $C_{n}$. Here $\Gamma_{1}$ and $\Gamma_{2}$ are attached to $c_{1}$ and $c_{2}$ respectively. $c_{3}$ is attached with $\Gamma_{3}, \Gamma_{4} ; c_{4}$ is attached with $\Gamma_{5}, \Gamma_{6}$ and so on. Tables 1 to 4 show the edge partition of $C_{n}^{R_{m}}$ :

\begin{tabular}{c|c|c|c|c}
\multicolumn{6}{c}{ Table 1. Edge partition of $c_{1}, c_{2}, \ldots ., c_{n}}$. \\
$\boldsymbol{R}_{(\boldsymbol{a}, \boldsymbol{b})}$ & $R_{(8,8)}$ & $R_{(8,11)}$ & $R_{(11,12)}$ & $R_{(12,12)}$ \\
\hline$\left|\boldsymbol{R}_{(\boldsymbol{a}, \boldsymbol{b})}\right|$ & 1 & 2 & 2 & $n-5$
\end{tabular}

Table 2. Edge partition of $c_{1}, c_{2}, \ldots, c_{n}$ with $R_{1}\left(\Gamma_{1}\right), R_{1}\left(\Gamma_{2}\right), \ldots, R_{1}\left(\Gamma_{(2 n-2)}\right)$.

\begin{tabular}{c|c|c|c}
$\boldsymbol{R}_{(\boldsymbol{a}, \boldsymbol{b})}$ & $R_{(8,5)}$ & $R_{(11,6)}$ & $R_{(12,6)}$ \\
\hline$\left|\boldsymbol{R}_{(\boldsymbol{a}, \boldsymbol{b})}\right|$ & 2 & 4 & $2(n-4)$
\end{tabular}

Table 3. Edge partition of $\Gamma_{1}$ and $\Gamma_{2}$.

\begin{tabular}{c|c|c|c|c|c|c}
$\boldsymbol{R}_{(\boldsymbol{a}, \boldsymbol{b})}$ & $R_{(5,6)}$ & $R_{(6,6)}$ & $R_{(6,3)}$ & $R_{(5,3)}$ & $R_{(6,3)}$ & $R_{(3,3)}$ \\
\hline$\left|\boldsymbol{R}_{(\boldsymbol{a}, \boldsymbol{b})}\right|$ & 2 & $2(m-3)$ & 2 & 4 & $4(m-2)$ & 6
\end{tabular}

Table 4. Edge partition of $\Gamma_{3, \ldots \ldots \ldots \ldots,} \Gamma_{(2 n-2)}$.

$$
\begin{aligned}
& \begin{array}{c|c|c|c|c}
\boldsymbol{R}_{(\boldsymbol{a}, \boldsymbol{b})} & R_{(6,6)} & R_{(6,3)} & R_{(6,3)} & R_{(3,3)} \\
\hline\left|\boldsymbol{R}_{(\boldsymbol{a}, \boldsymbol{b})}\right| & (m-2)(2 n-4) & 2 n-4 & 2(2 n-4)(m-1) & 3(2 n-4)
\end{array} \\
& R N M_{1}^{*}(G)=\sum_{u v \in E(G)}\left[d_{N^{\prime}}(u)+d_{N^{\prime}}(v)\right] \\
& =16+2(19)+2(23)+(n-5)(24)+2(13)+4(17)+2(n-4)(18)+2(11) \\
& +2(m-3)(12)+2(9)+4(8)+4(m-2)(9)+6(6)+(m-2)(2 n-4)(12) \\
& +(2 n-4)(9)+2(2 n-4)(m-1)(9)+3(2 n-4)(6) \\
& =60 n m+30 n-60 m-46 \text {. }
\end{aligned}
$$$$
\operatorname{RNM}_{3}^{*}(G)=\sum_{u v \in E(G)}\left[d_{N^{\prime}}(u)^{2}+d_{N^{\prime}}(v)^{2}\right]
$$$$
=128+370+530+(n-5) 288+178+628+2(n-4)(180)+122
$$$$
+2(m-3)(72)+90+136+4(m-2)(45)+108
$$$$
+(m-2)(2 n-4)(72)+(2 n-4)(45)+2(2 n-4)(m-1)(45)
$$$$
+3(2 n-4)(18)
$$ 


$$
=324 n m+378 n-324 m-842 .
$$

Theorem 6. Let $G \cong C_{n}^{R_{t}}$ be a molecular graph of a special type of cycloalkyne with $\mathrm{n} \geq 5$ and $t \geq 2$ vertices. Then

(i) $R N M_{1}^{*}(G)=60 n t+6 n-120 t-12$.

(ii) $R N M_{3}^{*}(G)=324 n t-54 n-648 t+12$.

Proof. Let $G \cong C_{n}^{R_{t}}$ be a molecular graph of a special type of cycloalkyne, as shown in Figure 5. Then the graph $G$ has $6 n t+3 n-12 t-4$ vertices of which $n$ of them represent the vertices of a cycle and $(2 n-4)(3 t+1)$ represent the vertices of alkyls. Out of these vertices, there are 4 vertices of reduced neighborhood degree $4, n-4$ vertices of reduced neighborhood degree $6,(2 n-4)(t-1)$ vertices of reduced neighborhood degree 6 and $(2 n-4)(2 t+2)$ vertices of reduced neighborhood degree 3 . Tables 5 and 6 show the edge partition of $C_{n}^{R_{t}}$ :

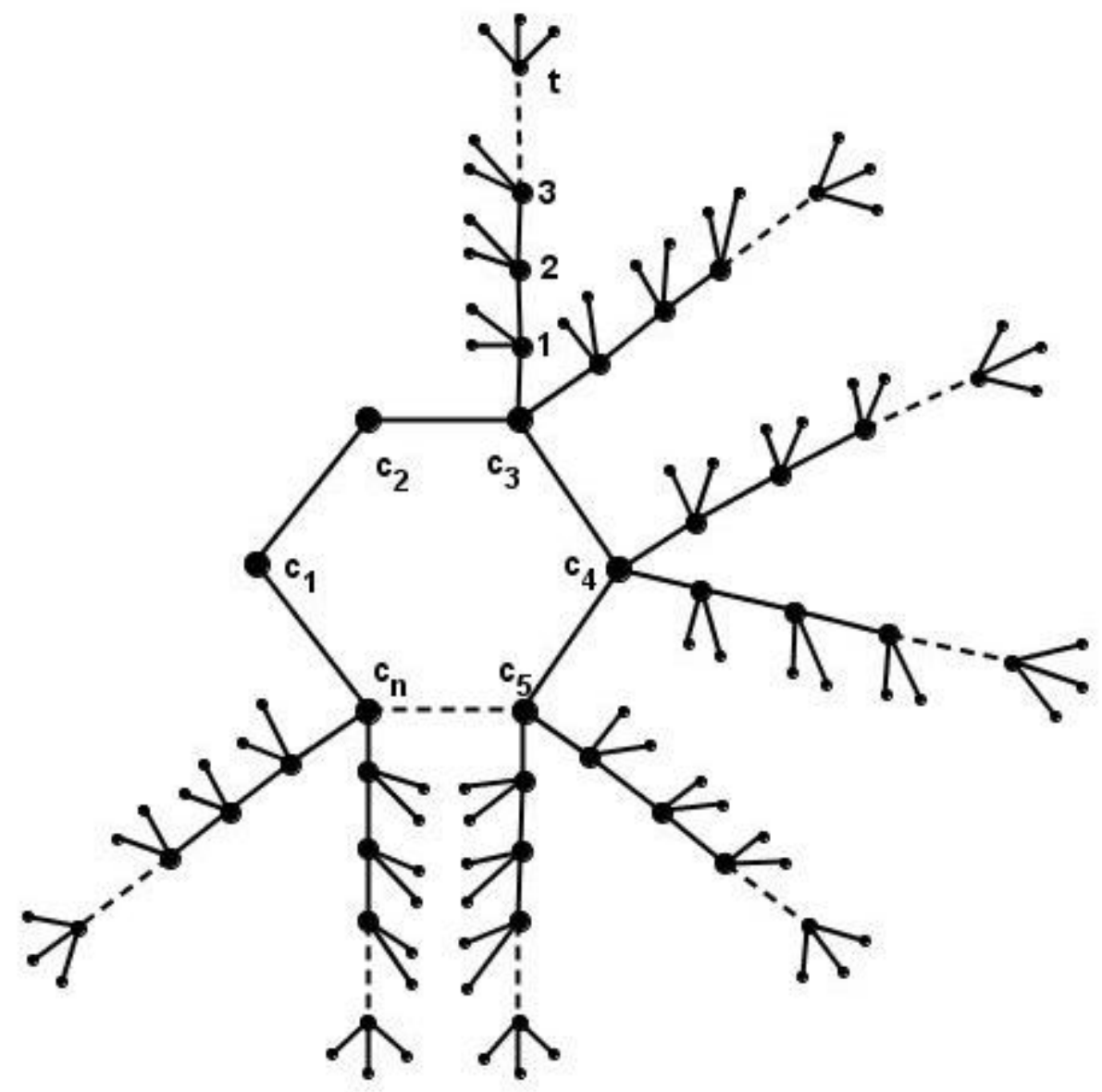

Figure 5. Molecular graph of $C_{n}^{R_{t}}$.

Table 5. Edge partition of a cycle.

\begin{tabular}{c|c|c|c} 
& $R_{(4,4)}$ & $R_{(4,6)}$ & $R_{(6,6)}$ \\
\hline $\boldsymbol{R}_{(\boldsymbol{a}, \boldsymbol{b})}$ & 2 & 2 & $n-5$
\end{tabular}

Table 6. Edge partition of alkyls.

\begin{tabular}{c|c|c|c|c}
$\boldsymbol{R}_{(\boldsymbol{a}, \boldsymbol{b})}$ & $R_{(4,6)}$ & $R_{(6,6)}$ & $R_{(6,3)}$ & $R_{(3,3)}$ \\
\hline$\left|\boldsymbol{R}_{(\boldsymbol{a}, \boldsymbol{b})}\right|$ & 4 & $(t-2)(2 n-4)+(2 n-8)$ & $(2 t-1)(2 n-4)$ & $3(2 n-4)$
\end{tabular}




$$
\begin{aligned}
R N M_{1}^{*}(G)= & \sum_{u v \in E(G)}\left[d_{N^{\prime}}(u)+d_{N^{\prime}}(v)\right] \\
= & 24+20+(n-5)(12)+40+[(t-2)(2 n-4)+(2 n-8)](12) \\
& +(2 t-1)(2 n-4)(9)+3(2 n-4)(6) \\
= & 60 n t-120 t+6 n-12 . \\
R N M_{3}^{*}(G)= & \sum_{u v \in E(G)}\left[d_{N^{\prime}}(u)^{2}+d_{N^{\prime}}(v)^{2}\right] \\
= & 96+104+(n-5) 72+208+[(t-2)(2 n-4)+(2 n-8)](72) \\
& +((2 t-1)(2 n-4))(45)+3(2 n-4)(18) \\
= & 324 n t-54 n-648 t+12 .
\end{aligned}
$$

\begin{tabular}{|c|c|c|c|c|c|c|c|c|}
\hline \multicolumn{3}{|c|}{$G_{1}$} & \multicolumn{3}{|c|}{$G_{2}$} & \multicolumn{3}{|c|}{$G_{3}$} \\
\hline Cycloalkanes & $R N M_{1}^{*}$ & $R_{N} M_{3}^{*}$ & Cycloalkenes & $R N M_{1}^{*}$ & $R N M_{3}^{*}$ & Cycloalkynes & $R N M_{1}^{*}$ & $R_{N} M_{3}^{*}$ \\
\hline Cyclopropane & 90 & 486 & Cyclopropene & 56 & 240 & Cyclopropyne & 30 & 98 \\
\hline Cyclobutane & 120 & 648 & Cyclobutene & 86 & 394 & Cyclobutyne & 60 & 228 \\
\hline Cyclopentane & 150 & 810 & Cyclopentene & 116 & 556 & Cyclopentyne & 90 & 390 \\
\hline Cyclohexane & 180 & 972 & Cyclohexene & 146 & 718 & Cyclohexyne & 120 & 552 \\
\hline Cycloheptane & 210 & 1134 & Cycloheptene & 176 & 880 & Cycloheptyne & 150 & 714 \\
\hline Cyclooctane & 240 & 1296 & Cyclooctene & 206 & 1042 & Cyclooctyne & 180 & 876 \\
\hline Cyclononane & 270 & 1458 & Cyclononene & 236 & 1204 & Cyclononyne & 210 & 1038 \\
\hline Cyclodecane & 300 & 1620 & Cyclodecene & 266 & 1366 & Cyclodecyne & 240 & 1200 \\
\hline
\end{tabular}

\subsection{QSPR analysis of modified, reduced neighborhood topological indices.}

In this section, we check the correlation coefficient of the newly defined indices to check their efficiency. For this purpose, we consider the molecular graphs of cycloalkanes, cycloalkenes, and cycloalkynes of order $n \leq 10$ and divide them into three classes as $G_{1}, G_{2}, G_{3}$ respectively as shown in 7 .

Table 7. Modified reduced neighborhood topological index values.

Table 8. Correlation Coefficients.

\begin{tabular}{l|l|l|c|c|c}
$\boldsymbol{R} N \boldsymbol{M}_{\mathbf{1}}^{*}\left(\boldsymbol{G}_{\mathbf{1}} \& \boldsymbol{G}_{\mathbf{2}}\right)$ & 1 & $\boldsymbol{R} \boldsymbol{N} \boldsymbol{M}_{\mathbf{3}}^{*}\left(\boldsymbol{G}_{\mathbf{1}} \& \boldsymbol{G}_{\mathbf{2}}\right)$ & 0.999983 & $\boldsymbol{G}_{\mathbf{1}}: \boldsymbol{R} \boldsymbol{N} \boldsymbol{M}_{\mathbf{1}}^{*} \& \boldsymbol{R} \boldsymbol{N} \boldsymbol{M}_{\mathbf{3}}^{*}$ & 1 \\
\hline $\boldsymbol{R} \boldsymbol{N} \boldsymbol{M}_{\mathbf{1}}^{*}\left(\boldsymbol{G}_{\mathbf{1}} \& \boldsymbol{G}_{\mathbf{3}}\right)$ & 1 & $\boldsymbol{R} \boldsymbol{N} \boldsymbol{M}_{\mathbf{3}}^{*}\left(\boldsymbol{G}_{\mathbf{1}} \& \boldsymbol{G}_{\mathbf{3}}\right)$ & 0.99972 & $\boldsymbol{G}_{\mathbf{2}}: \boldsymbol{R} \boldsymbol{N} \boldsymbol{M}_{\mathbf{1}}^{*} \& \boldsymbol{R} \boldsymbol{N} \boldsymbol{M}_{\mathbf{3}}^{*}$ & 0.999983 \\
\hline $\boldsymbol{R} \boldsymbol{N} \boldsymbol{M}_{\mathbf{1}}^{*}\left(\boldsymbol{G}_{\mathbf{2}} \& \boldsymbol{G}_{\mathbf{3}}\right)$ & 1 & $\boldsymbol{R} \boldsymbol{N} \boldsymbol{M}_{\mathbf{3}}^{*}\left(\boldsymbol{G}_{\mathbf{2}} \& \boldsymbol{G}_{\mathbf{3}}\right)$ & 0.999841 & $\boldsymbol{G}_{\mathbf{3}}: \boldsymbol{R} \boldsymbol{N} \boldsymbol{M}_{\mathbf{1}}^{*} \& \boldsymbol{R} \boldsymbol{N} \boldsymbol{M}_{\mathbf{3}}^{*}$ & 0.99972
\end{tabular}

From Table 8, it can be observed that the indices $R N M_{1}^{*}$ and $R N M_{3}^{*}$ are highly correlated with respect to $G_{1}, G_{2}$ and $G_{3}$ and hence the modified, reduced neighborhood first Zagreb index and forgotten reduced neighborhood index can be considered novel topological indices.

\section{Conclusions}

In this research work, we have used the modified, reduced neighborhood topological indices to calculate the values of cycloalkanes, cycloalkenes, and cycloalkynes. We have defined a new family of molecular graphs by replacing the hydrogen atoms with alkyls in the above-mentioned molecular graphs. We have also examined the correlation coefficient of the newly defined indices to check their efficiency by considering the molecular graphs of order, $n \leq 10$. We find that $R N M_{1}^{*}\left(G_{1}\right.$ and $\left.G_{2}\right), R N M_{1}^{*}\left(G_{1}\right.$ and $\left.G_{3}\right)$ and $R N M_{1}^{*}\left(G_{2}\right.$ and $\left.G_{3}\right)$ have the 
highest correlation. Also, the correlation coefficient between $R N M_{1}^{*}$ and $R N M_{3}^{*}$ of the family of graphs in $G_{1}$ exhibits the stronger correlation. Hence these topological indices can be considered novel topological indices.

\section{Funding}

This research received no external funding.

\section{Acknowledgments}

The authors declare no acknowledgments.

\section{Conflicts of Interest}

The authors declare no conflict of interest.

\section{References}

1. Gutman, I.; Trinajstic, N.; Graph theory and molecular orbitals, Total $\pi$-electron energy of alternant Hydrocarbons. Chemical Physics Letters 1972, 17, 535-538, https://doi.org/10.1016/0009-2614(72)85099-1.

2. Alwardi, A.; Alqesmah, A.; Rangaranjan, R.; Cangul, I. N.; Entire Zagreb indices of graphs. Discrete Mathematics, Algorithms and Applications 2018, 10, 1850037, https://doi.org/10.1142/S1793830918500374.

3. Mondel, S.; Dey, A.; De, N.; Pal, A.; QSPR analysis of some novel neighborhood degree-based topological descriptors. Complex \& Intelligent Systems 2021, 7, 977-996, https://doi.org/10.1007/s40747-020-00262-0.

4. Zheng, L.; Wang, Y.; Gao, W.; Topological indices of hyaluronic acid-Paclitaxal conjugates molecular structure in cancer treatment. Open Chem, 2019, 17, 81-87, https://doi.org/10.1515/chem-2019-0009.

5. Gao, W.; Siddiqui, M.K.; Imran, M.; Jamil, M. K.; Farahani, M.R.; Forgotten topological index of chemical structure in drugs. Saudi pharmacetucal Journal 2016, 24, 258-264, https://doi.org/10.1016/j.jsps.2016.04.012.

6. Kulli, V.R.; Revan inidces of Chloroquine, Hydroxychloroquine, Remdesiver: Research advances for the treatment of COVID-19. Int. J. Eng. Sci. Technol. 2020, 9, 73-74, https://doi.org/10.5281/zenodo.3828871.

7. Basavanagoud, B.; Barangi, A.P.; Hosamani, S.M.; First neighborhood Zagreb index of some nanostructures. Proceedings of IAM 2018, 7, 178-193, https://doi.org/10.1145/509404.509415.

8. Ahmed, H.; Alwardi, A.; Ruby Salestina, M.; On domination topological indices of graphs. International journal of analysis and applications 2021, 19, 47-64, https://doi.org/10.28924/2291-8639-19-2021-47.

9. Ahmed, H.; Ruby Salestina, M.; Alwardi, A.; Domination topological indices and their polynomials of a firefly graph. Journal of discrete mathematical sciences and cryptography 2021, 24, 325-341, https://doi.org/10.1080/09720529.2021.1882155.

10. Ahmed, H.; Farahani, H.R.; Alwardi, A.; Ruby Salestina, M.; Domination topological properties of some chemical structures using $\varphi_{p}$ - polynomial approach. Euresian chemical communications 2021, 3, 210-218, https://doi.org/10.22034/ecc.2021.271992.1133.

11. Imran, M.; Naeem, M.; Baig, A.Q.; Topological indices of polyhydroxybutyrate and polycaprolactone. Journal of information and optimization sciences 2020, 41, 1025-1041, https://doi.org/10.1080/02522667.2020.1747192.

12. Mondal, S.; De, N.; Pal, A.; Topological Indices of Some Chemical Structures Applied for the Treatment of COVID-19 Patients, Polycycl. Aromat. Compd. 2020, https://doi.org/10.1080/10406638.2020.1770306.

13. Saleh, A.; Sophia Shalini, G.B., Dhananjayamurthy, B.V., The reduced neighborhood topological indices and RNM-polynomial for the treatment of COVID-19. Biointerface research in applied chemistry 2020, 11, 11817-11832, https://doi.org/10.33263/BRIAC114.1181711832.

14. Gao, W.; Chen, Y., Wang, W.; The topological variable computation for a special type of cycloalkanes. Journal of chemistry 2017, 2017, 6534758, https://doi.org/10.1155/2017/6534758.

15. Mathad, V.; Padmapriya, P.; Cangul, I.N.; Some topological indices of certain classes of cycloalkenes. Proceedings of the jangjoen mathematical society 2019, 22, 233-247, https://doi.org/10.17777/pjms2019.22.2.233.

16. Mohammed, M.A.; Atan, K.A.; Khalaf, A.M.; Hansi, R.; Mohammed Said, M.R.; Atom bond connectivity index of molecular graphs of alkynes and cycloalkynes. Journal of computational and theoretical nanoscience 2016, 13, 6698-6706, https://doi.org/10.1166/jctn.2016.5616.

17. El-Sayed, M.T.; Suzen, S.; Altanlar, N.; Ohlsen, K.; Hilgeroth, A.; Discovery of bisindolyl-substituted cycloalkane-anellated indoles as novel class of antibacterial agents against S. aureus and MRSA. Bioorganic and medical chemistry letter 2016, 26, 218-221, https://doi.org/10.1016/j.bmcl.2015.10.085. 
18. Denicourt-Nowicki, A.; Lebedeva, A.; Bellini, C.; Roucoux, A.; Highly selective cycloalkane oxidation in water with ruthenium nanoparticals. ChemCatChem 2015, 8, 357-362, https://doi.org/10.1002/cctc.201500805.

19. Becker, K.B.; Cycloalkenes by intermolecular witting reaction. Tetrahedron 1980, 36, 1717-1745, https://doi.org/10.1016/0040-4020(80)80068-8.

20. Saeed, N.; Long, K.; Mufti, Z.S.; Sajid, H.; Rehman, A.; Degree based topological indices of Boron $B_{12}$. Journal of Chemistry 2021, 2021, 5563218, https://doi.org/10.1155/2021/5563218.

21. Hong, G.; Gu, Z.; Javaid, M.; Awais, H.M.; Siddiqui, M.K.; Degree based topological indices of metal organic networks. IEEE Access 2020, 8, 68288-68300, https://doi.org/10.1109/ACCESS.2020.2985729.

22. Mondel, S.; Imran, M.; De, N.; Pal, A.; Neighborhood M-polynomial of titanium compounds. Arabian jounal of chemistry 2021, 14, 103244, https://doi.org/10.1016/j.arabjc.2021.103244. 